\title{
Left ventricular outflow obstruction in a patient with undiagnosed hypertrophic obstructive cardiomyopathy
}

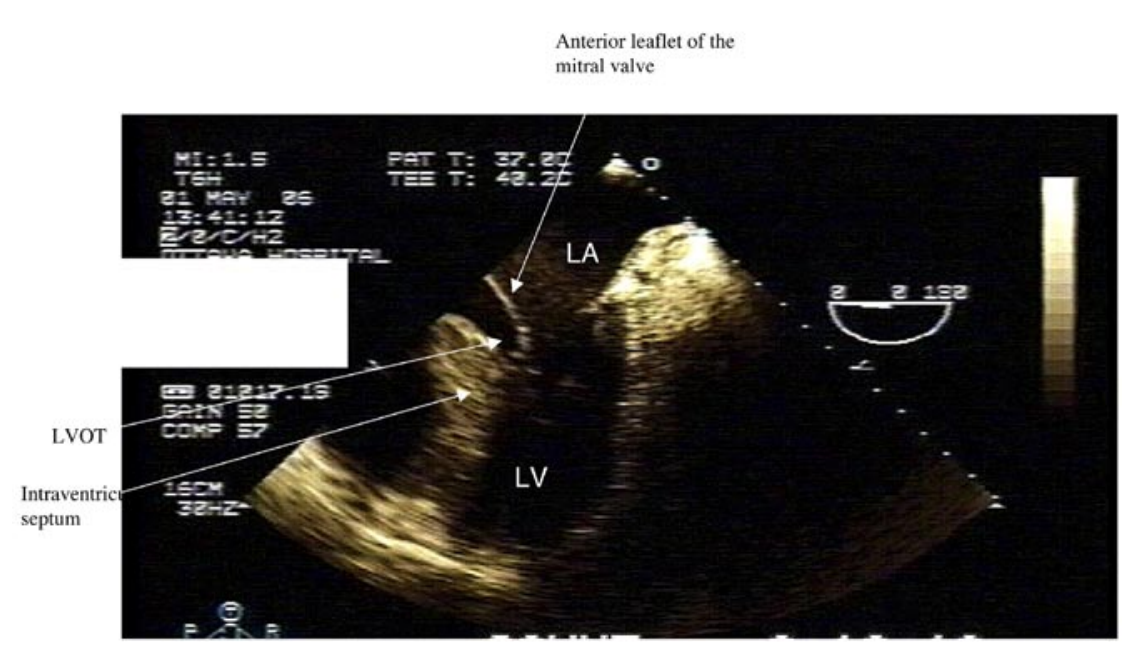

FIGURE 1 A mid-esophageal five-chamber view showing systolic anterior motion of the mitral valve with left ventricle outflow tract obstruction. LVOT = left ventricle outflow tract; LV = left ventricle; LA = left atrium.

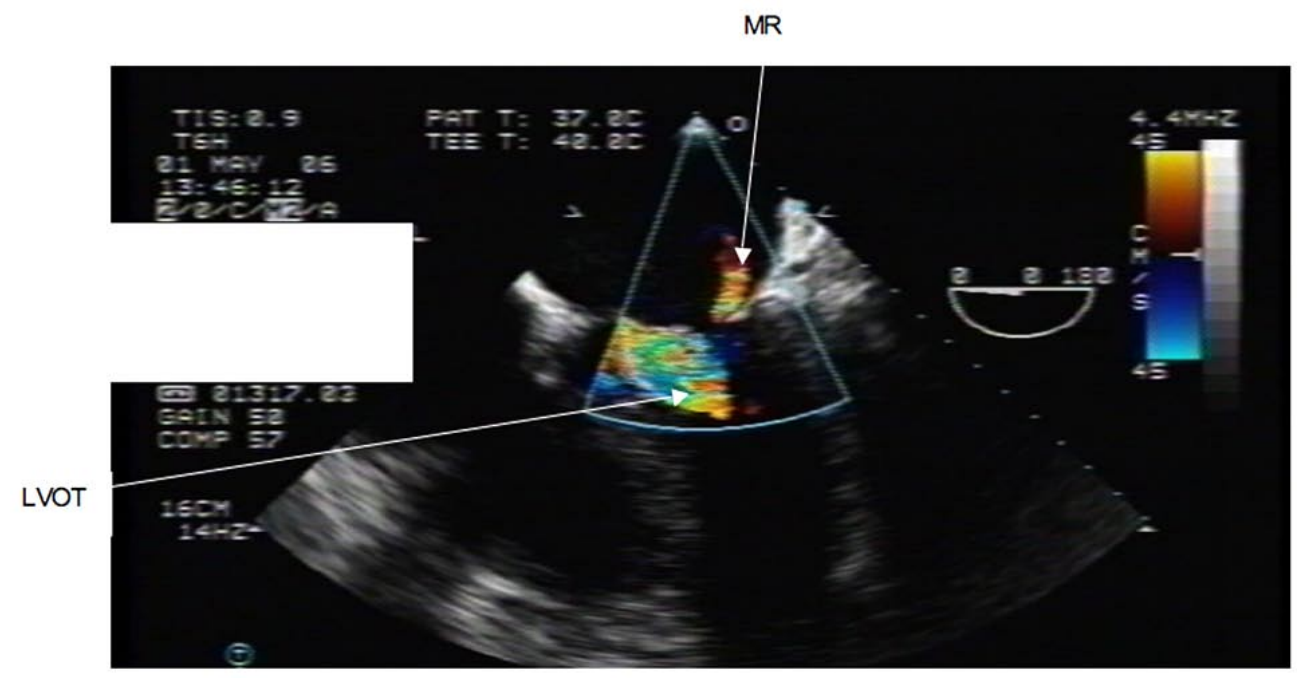

FIGURE 2 Colour flow Doppler of the left ventricle outflow tract (LVOT) at the mid-esophageal level with a five-chamber view. Note the posteriorly-directed mitral regurgitation (MR) and the turbulent flow at the LVOT. 


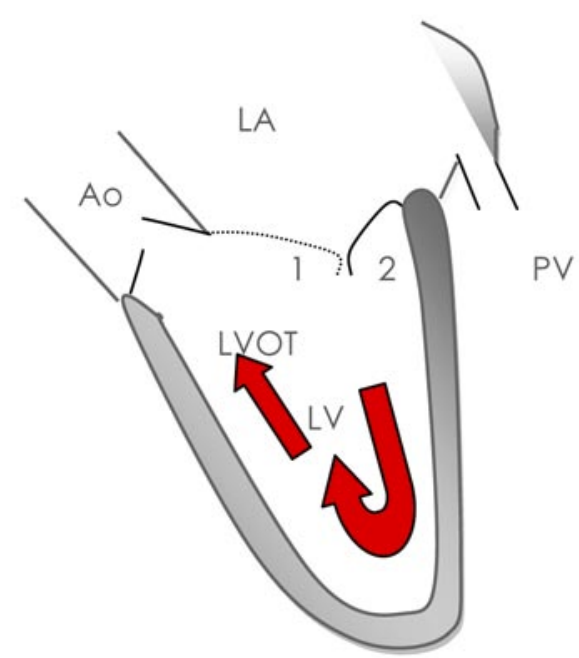

FIGURE 3 Left ventricular outflow tract (LVOT) of the normal heart. $\mathrm{LA}=$ left atrium, $\mathrm{LV}=$ left ventricle, $\mathrm{PV}=$ pulmonary vein, LVOT = left ventricular out flow tract, 1 $=$ anterior leaflet of the mitral valve, 2 = posterior leaflet of the mitral valve; Ao $=$ aorta .

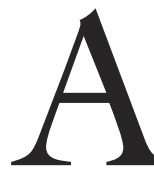

46-yr-old male patient with obstructive sleep apnea presented for an elective septoplasty. He had a longstanding history of hypertension and occasional episodes of shortness of breath during moderate exercise. There was no previous hospital admission related to cardiac or respiratory illness. He had no history of previous surgery and no known drug allergies. Physical examination revealed an overweight patient with a body mass index of $43 \mathrm{~kg} \cdot \mathrm{m}^{-2}$. Heart sounds were muffled and the remainder of the physical examination was unremarkable. His medications included atenolol 50 mg PO bid and multi-vitamins.

Following induction of anesthesia, the patient's blood pressure decreased to $65 / 30 \mathrm{mmHg}$. Incremental doses of ephedrine did not prevent a further decrease in blood pressure. Epinephrine $50 \mu \mathrm{g}$ iv resulted in tachycardia (heart rate 102 beats. $\mathrm{min}^{-1}$ ) without any improvement in blood pressure. A transesophageal echocardiography assessment was immediately undertaken. A mid-esophageal five-chamber view revealed systolic anterior motion of the anterior leaflet of the mitral valve (Figure 1). This resulted in left ventricular outflow obstruction (Figure 2). The patient was treated with intravenous fluids, esmolol and phenylephrine, which resulted in hemodynamic improvement. Surgery was postponed and the patient was referred to cardiology for further evaluation.

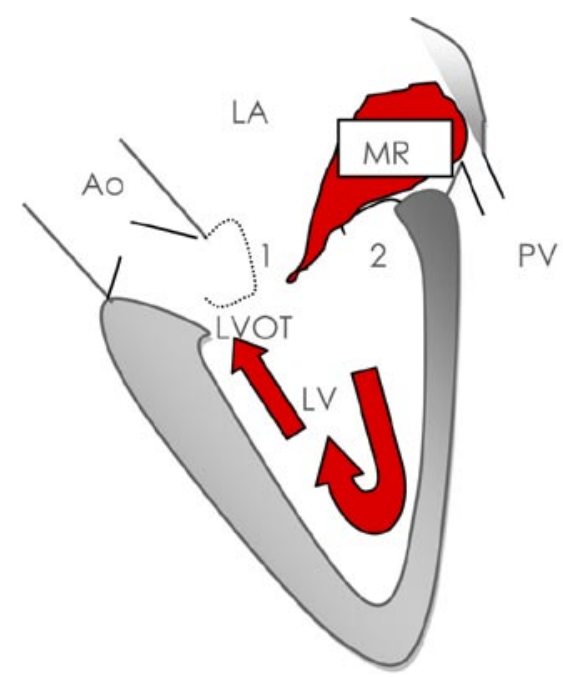

FIGURE 4 Left ventricular outflow tract (LVOT) obstruction caused by the anterior leaflet of the mitral valve (1). The patient had intraventricular septal hypertrophy and mitral regurgitation $(\mathrm{MR})$ directed posteriorly. $\mathrm{LA}=\mathrm{left}$ atrium, LV = left ventricle, PV = pulmonary vein, LVOT = left ventricular out flow tract, $1=$ anterior leaflet of the mitral valve, 2 = posterior leaflet of the mitral valve; $M R=$ mitral regurgitation.

\section{Ashraf Fayad MD FASE FCARCSI}

The Ottawa Hospital, University of Ottawa, Ottawa, Canada

E-mail: afayad@ottawahospital.on.ca

Accepted for publication September 26, 2007.

\section{Reference}

1 Anonymous. Practice guidelines for perioperative transesophageal echocardiography. A report by the American Society of Anesthesiologists and the Society of Cardiovascular Anesthesiologists Task Force on Transesophageal Echocardiography. Anesthesiology 1996: 84: 986-1006. 